\section{Eficiência dos estados brasileiros e do Distrito Federal no sistema público de transplante renal: uma análise usando método DEA (Análise Envoltória de Dados) e índice de Malmquist}

\author{
Efficiency of Brazilian States and the Federal \\ District in the public kidney transplant system \\ based on DEA (Data Envelopment Analysis) \\ and the Malmquist index
}

\author{
Eficiencia de los estados brasileños y el Distrito \\ Federal en el sistema público de trasplante de \\ riñón: un análisis utilizando el procedimiento \\ DEA (Análisis de Involucrados de Datos) e \\ índice Malmquist
}

\begin{abstract}
${ }^{1}$ Centro de Ensino Superior de Maringá, Maringá, Brasil. 2 Universidade Federal do Rio Grande do Sul, Porto Alegre, Brasil.

3 Universidade Federal do Rio Grande do Norte, Natal, Brasil.

Correspondência C. K. F. Costa Centro de Ensino Superior de Maringá.

Av. Guedner 1610, Maringá, PR 87050-390, Brasil. cfavoretto@hotmail.com
\end{abstract}

Abstract
The objective of this study was to evaluate the efficiency of Brazilian States and the Federal District in the public kidney transplant system and their productivity trends from 2006 to 2011. The authors used Data Envelopment Analysis (DEA) with slack and the Malmquist index with slack. Inputs included spending on hospital services and health professionals in the system. The output was the number of kidney transplants performed in each State. The data showed a significant discrepancy between States in the number of kidney transplants. The transplant system's inefficiency may result from inadequate management, failure to comply with national guidelines, inactive hospital transplant committees, and overburdened hospital staff. Institutional changes promoted by the Brazilian Ministry of Health (procedures improvement and standardization) failed to increase productivity in most States during this period.

Kidney Transplantation; Health Management; Unified Health System
Cassia Kely Favoretto Costa 1

Giácomo Balbinotto Neto 2

Luciano Menezes Bezerra Sampaio ${ }^{3}$

\section{Resumo}

O objetivo foi avaliar a eficiência dos estados brasileiros e do Distrito Federal no sistema público de transplante renal e a mudança de produtividade deles entre 2006 e 2011. Utilizou-se a Análise Envoltória de Dados (DEA) baseada em folgas e o índice de Malmquist com folgas. Os inputs usados foram os gastos dos serviços hospitalares e dos profissionais no sistema. Como output, o número de transplantes renais realizados por cada estado. Existe uma discrepância significativa em relação à captação e ao número de transplantes renais entre os estados. Evidencia-se uma ineficiência nesse sistema, que poder ser causada por sua gestão inadequada; não seguimento de regras nacionais; comissões intra-hospitalares não ativas e equipes hospitalares sobrecarregadas. As mudanças institucionais promovidas pelo Ministério da Saúde (aperfeiçoamento e padronização dos processos) não geraram aumento de produtividade na maioria dos estados nesse período.

Transplante Renal; Gestão em Saúde; Sistema Único de Saúde 


\section{Introdução}

No período recente, o Brasil possui o maior sistema público de transplantes de órgãos do mundo e ocupa o segundo lugar (atrás apenas dos Estados Unidos) no número (absoluto) de cirurgias desse porte, com destaque para o transplante renal $1,2,3,4,5,6,7,8$.

No Brasil, o Sistema Único de Saúde (SUS) é responsável por todos os custos dos transplantes renais, desde a busca do potencial doador até o acompanhamento do paciente após o transplante $9,10,11$. Os gastos totais do SUS com procedimentos hospitalares relacionados aos transplantes renais passaram de $\mathrm{R} \$ 35,63$ milhões, em 2000, para R \$ 85,4 milhões, em 2010 - crescimento de $139,89 \%$ (Sistema Nacional de Transplantes, Departamento de Informática do SUS. http:// snt.datasus.gov.br/SNT/index.jsf, acessado em 10/Jun/2011. Departamento de Informática do SUS. Procedimentos Hospitalares do SUS - Por Local de Internação - Brasil. http://tabnet.data sus.gov.br/cgi/deftohtm.exe?sih/cnv/qiuf.def, acessado em 11/Jun/2011). Apesar dessa expansão, existe um significativo desequilíbrio entre a oferta e a demanda por rim no país que leva ao surgimento de filas e aumento no tempo de espera por um transplante 12,13,14,15. Esse desequilíbrio pode estar associado ao envelhecimento da população e, consequentemente, ao agravamento das doenças renais crônicas 16,17,18. Além disso, existem discrepâncias no processo de captação e transplante desse órgão entre os estados brasileiros 19,20,21.

O Ministério da Saúde, desde 1997, vem buscando adotar medidas legais, gerenciais e administrativas para tornar o funcionamento do setor de transplantes mais eficiente. Uma das principais ações foi a criação da Política Nacional dos Transplantes (Lei no 9.434, de 4 de fevereiro) 22 . Essa lei estabeleceu os critérios sobre a remoção de órgãos, tecidos e partes do corpo humano direcionados para transplantes 22,23. Para regulamentar a gestão administrativa prevista nessa lei, o Decreto no 2.268 24, de 30 de junho de 1997, instituiu o Sistema Nacional de Transplantes (SNT) junto a esse ministério, para realizar o processo de captação e distribuição de órgãos e tecidos 25 .

Ao longo da história dos transplantes de órgãos e tecidos, outras leis importantes ( $L e i$ no 10.211 26, de 21 de março de 2001 e Lei no 11.52127 , de 18 de setembro de 2007) foram implementadas para melhorar o desempenho do SNT; contudo, até recentemente, foram identificados problemas de ineficiência ${ }^{21}$ nas atividades de gestão, gerenciamento, execução, controle e monitoramento das ações desse sistema 28.
Em 21 de outubro de 2009, o Ministério da Saúde implementou a Portaria no 2.600 29, aprovando o novo Regulamento Técnico do SNT. A portaria, além de atualizar as leis que fundamentavam o sistema até aquele momento, aperfeiçoou e padronizou o funcionamento do sistema. As principais mudanças foram as seguintes: (a) estabeleceram normas específicas para a autorização de funcionamento dos órgãos gestores do SNT, dos estabelecimentos de saúde e das equipes especializadas; (b) houve o aprimoramento dos mecanismos de funcionamento e gerenciamento do SNT, das Centrais de Notificação, Captação e Distribuição de Órgãos (CNCDO) e dos demais integrantes do sistema, de forma a permitir melhor articulação entre essas instâncias; e (c) aperfeiçoaram as normas e o processo de supervisão, gestão e controle das listas de espera de potenciais receptores (estaduais, regionais e nacional), buscando garantir a equidade e a transparência na distribuição de órgãos e tecidos para transplantes 29 .

De forma geral, no atual SNT, todos os órgãos e tecidos captados são distribuídos segundo o método de Lista Única, ou seja, seguem um conjunto de critérios específicos de distribuição, selecionando o receptor mais adequado. A Coordenação-Geral do SNT (CGSNT) é responsável pela normatização e regulamentação dos procedimentos relacionados à captação, alocação e distribuição de órgãos; pelo credenciamento das CNCDO e pela autorização dos estabelecimentos de saúde e equipes especializadas a realizar retiradas, transplantes ou enxertos de tecidos, órgãos e partes do corpo (Sistema Nacional de Transplantes, Departamento de Informática do SUS. http://snt.datasus.gov.br/SNT/index.jsf, acessado em 10/Jun/2011).

Em conjunto com a CGSNT, atua a Central Nacional de Transplante (ou Central Nacional de Notificação, Captação e Distribuição de Órgãos - CNNCDO), situada no Aeroporto de Brasília, funcionando 24 horas por dia. A CGSNT e a CNNCDO são classificadas como participantes da estância normativa do SNT.

As CNCDO são classificadas como unidades executivas estaduais do SNT. Tais órgãos têm a função de coordenar as atividades do transplante nesse nível, fazendo as inscrições e classificação dos receptores. Além disso, ao receber a notificação do diagnóstico de morte encefálica de um potencial doador, deve providenciar o transplante do órgão doado até o local em que será realizado o transplante (Sistema Nacional de Transplantes, Departamento de Informática do SUS. http://snt.datasus.gov.br/SNT/index.jsf, acessado em 10/Jun/2011). 
Em nível regional, estão as Comissões Intrahospitalares para Doação de Órgãos e Tecidos para Transplantes (CIHDOTT) cujas principais funções são as seguintes: (a) organizar, no âmbito do hospital, o processo de captação de órgãos; (b) articular-se com as equipes médicas das Unidades de Terapia Intensiva (UTI); (c) realizar a identificação e manutenção de potenciais doadores; (d) coordenar a abordagem familiar do potencial doador; e (e) articular-se com o Instituto Médico-Legal sobre o processo de necropsia dos doadores 30 .

Em parceria com as CIHDOTT, estão sendo implantadas no Brasil as Organizações de Procura de Órgãos e Tecidos (OPO) - já existentes no Estado de São Paulo -, que apresentam a função de coordenação supra-hospitalar e são responsáveis por organizar e apoiar as atividades relacionadas ao processo de doação de órgãos e tecidos 31,32 .

Nesse contexto, a eficiência na captação de rim para transplantes tem sido uma das preocupações centrais do SNT e dos formuladores de políticas públicas. A metodologia Análise Envoltória de Dados (DEA) vem sendo empregada para estimar a eficiência no setor de saúde, inclusive no setor de transplantes de órgãos e tecidos 4,19,33. Esse método é um instrumento robusto de tomada de decisão e de apoio ao monitoramento dessas políticas 34 . Portanto, torna-se relevante o desenvolvimento de uma avaliação sistemática do SNT, que permita a otimização do seu desempenho e, com isso, o efetivo gerenciamento do programa, da lista única de espera e a possível expansão da oferta do órgão rim para transplantes.

Diante desse contexto, este estudo tem os seguintes objetivos: (a) analisar a eficiência dos estados brasileiros e do Distrito Federal no sistema público de transplante renal, nos anos de 2006 e 2011; e (b) avaliar o desempenho da eficiência dos estados e do Distrito Federal ao longo do período de tempo em tela. Buscou-se, assim, analisar o comportamento dos estados e do Distrito Federal nesse setor, antes e após as mudanças institucionais adotadas pelo SUS, por meio da Portaria no 2.60029 .

\section{Material e método}

Nessa seção, estão apresentadas as técnicas DEA baseado em folgas (Slack Based Measure - SBM) e índice de Malmquist (Malmquist index - MI) com folgas, além da fonte de dados do estudo e das variáveis inputs (insumo) e output (produto) escolhidas para análise.
A metodologia DEA é um método não paramétrico que busca construir as fronteiras de eficiência por meio da programação linear, não necessitando da especificação de relações funcionais entre input e output. A DEA determina a eficiência com a qual uma unidade produtiva (unidade tomadora de decisão - decision making units - DMU) converte o input em output, na comparação com outras unidades analisadas 34 .

O modelo original da DEA foi desenvolvido por Charnes et al. 35 , sob a hipótese de retorno constante de escala (modelo CCR ou CRS - Constant Return to Scale) e, em seguida, modificado por Banker et al. 36 para possibilitar retorno variável de escala (modelo BCC ou VRS - variable return to scale). Nesse modelo, os fatores de escala são classificados em: (a) retornos crescentes de escala, quando as DMU operam com baixos valores de inputs; (b) retornos decrescentes de escala, ao admitir que as DMU trabalhem com altos valores de inputs; e (c) retornos constantes, que ocorrem no ponto em que a transição do primeiro para o segundo tipo de rendimento é realizada 34 .

A apresentação da metodologia empírica usada nesta pesquisa tem como base os estudos Cooper et al. 37 ; Ferreira \& Gomes 38 e Tone 39,40 . Nesse contexto, utilizou-se o modelo DEA baseado em folgas. Na DEA, o formato da fronteira não paramétrica linear pode gerar dificuldades na mensuração da eficiência das DMU, vale dizer, podem existir aquelas que sejam classificadas como eficientes, mas em razão das folgas (sla$c k s$ ), são avaliadas como falso eficiente. As folgas são excessos de insumos ou escassez de produtos utilizados por alguma DMU que pode estar numa parte da fronteira não eficiente por causa dessas folgas. Portanto, o modelo DEA com folga foi aplicado para que as DMU sejam realmente eficientes 39,40 .

Marinho \& Cardoso 19 e Marinho 33 sugerem que, no sistema de transplantes, os modelos de eficiência devem ser orientados para os outputs (isto é, maximizar os produtos sem diminuir os insumos), pois os gestores do sistema buscam a expansão dos recursos financeiros disponíveis e da quantidade de transplantes renais realizados. Este trabalho segue a mesma orientação.

\section{Modelo DEA-SBM}

Para estimar a eficiência dos estados brasileiros e do Distrito Federal no sistema público de transplante renal, adotou-se o modelo DEA baseado em folgas (DEA-SBM), proposto por Tone 39,40 . A estimação da eficiência de uma DMU $\left(x_{0}, y_{0}\right)$ é obtida resolvendo-se o seguinte problema de programação linear: 
$\operatorname{Min}_{\lambda, s^{-}, s^{+}} \rho=\frac{1-\frac{1}{m} \sum_{i=1}^{m} s_{i}^{-} / x_{i 0}}{1+\frac{1}{s} \sum_{i=1}^{m} s_{r}^{+} / y_{r 0}}$

$$
\text { Sujeito a: } \begin{aligned}
& x_{0}=X \lambda+s^{-} \\
& y_{0}=Y \lambda-s^{+} \\
& \lambda \geq 0, s^{-} \geq 0, s^{+} \geq 0
\end{aligned}
$$

Na equação (1), $\lambda$ corresponde ao peso dos inputs e outputs, e $\rho$ são os vetores de folgas de cada um deles, respectivamente. No modelo, deve-se considerar a seguinte condição: $0 \leq \rho \leq 1$, onde $\rho$ corresponde à mensuração da eficiência das DMU (estados brasileiros e Distrito Federal). Quando $\rho=1$, as DMU são consideradas eficientes ou ineficientes e com folgas. Assim, unidades ineficientes têm $\rho<1$ se estiverem abaixo da fronteira ou podem estar sobre a fronteira ( $\operatorname{com} \rho=1$ ) e apresentarem folgas 37,39,40.

Nessa metodologia, admitem-se duas propriedades: (a) a mensuração é invariável (constante) em relação à unidade de análise de cada input e output, ou seja, o numerador e denominador da equação (1) são medidos na mesma unidade; e (b) em cada folga de input e output a mensuração é classificada como monotônica e decrescente, isto é, o valor da função objetivo reduz após aumentos em ${s_{i}}^{-}$e ${s_{r}}^{+}$, mantendo constantes os demais termos 37 .

No modelo DEA-SBM é assumida uma orientação ao input, ao output e também nenhuma orientação. Utiliza-se nesta pesquisa o modelo DEA-SBM orientado ao output, que pode ser expresso pela equação (2):

$$
\begin{array}{r}
\rho_{0}^{*}=\min _{\lambda, s^{+}} \frac{1}{1+\frac{1}{s} \sum_{r=1}^{s} s} \\
x_{0} \geq X \lambda
\end{array}
$$

Sujeito a: $y_{0}=Y \lambda-s^{+}$

$$
\lambda \geq 0, s^{+} \geq 0
$$

\section{Método de Malmquist-DEA com folgas}

Para comparar os escores de eficiência ao longo do tempo, é usado o MI com folga. Esse método foi desenvolvido por Färe et al. ${ }^{41}$ para medir o desempenho da produtividade das DMU entre dois períodos de tempo distintos.

O MI procura captar dois tipos de efeitos: (a) emparelhamento (catch-up effect), no qual se analisa o aumento ou redução da eficiência técnica produtiva ao longo do tempo; e (b) deslocamento da fronteira eficiente (frontier-shift effect), que reflete os avanços na produtividade de DMU, devido às inovações tecnológicas (ou ainda, gerenciais, legais e administrativas, como é o caso aqui analisado) 37,38 .

Para analisar o efeito emparelhamento (EEMP), admitem-se as notações de insumo (x) e produto (y), $\left(x_{o}, y_{o}\right)^{t}=\left(x_{o}{ }^{t}, y_{o}{ }^{t}\right)$ e $\left(x_{o}, y_{o}\right)^{t+1}=\left(x_{o}{ }^{t+1}, y_{o}{ }^{t+1}\right)$ para dois períodos $\mathrm{de}$ tempo, $\mathrm{t}$ e $\mathrm{t}+1$. O efeito do período $t+1$ para o período $t$ é mensurado da seguinte maneira:

Emparelhamento $=$

$\frac{\text { Eficiencia de }\left(x_{o}, y_{o}\right)^{t+1} \text { em relação a fronteira período } t+1}{\text { Eficiência de }\left(x_{o}, y_{o}\right)^{t} \text { em relação a fronteira período } t}$

Ou ainda, admitindo que as notações $\delta^{t+1}\left(\left(x_{0} ; y_{0}\right)^{t+1}\right)$ e $\delta^{t}\left(\left(x_{0} ; y_{0}\right)^{t}\right)$ representam o escore de eficiência das DMUs no período $t+1$ e $t$, a equação (3) pode ser expressa como:

Emparelhamento $=\frac{\delta^{t+1}\left(\left(x_{o}, y_{o}\right)^{t+1}\right)}{\delta^{t}\left(\left(x_{o}, y_{o}\right)^{t}\right)}$

A análise da equação 3.1 é feita sob três óticas: (a) se o emparelhamento é maior que 1 , existe melhora na eficiência técnica entre os períodos $t$ e $t+1$; (b) se o emparelhamento é igual a 1 , indica que a eficiência permaneceu a mesma; e (c) no caso, se o emparelhamento for menor que 1 , representa piora da eficiência.

Por sua vez, o efeito deslocamento da fronteira é representado da seguinte forma:

$F=\left[\frac{\delta^{t}\left(\left(x_{o}, y_{o}\right)^{t}\right)}{\delta^{t+1}\left(\left(x_{o}, y_{o}\right)^{t}\right)} \times \frac{\delta^{t}\left(\left(x_{o}, y_{o}\right)^{t+1}\right)}{\delta^{t+1}\left(\left(x_{o}, y_{o}\right)^{t+1}\right)}\right]^{1 / 2}$

Multiplicando a equação (3.1) por (4) e realizando operações algébricas, obtém-se o MI orientado ao produto (enfoque desse estudo), dado por:

$M I=\left[\frac{\delta^{t}\left(\left(x_{o}, y_{o}\right)^{t+1}\right)}{\delta^{t}\left(\left(x_{o}, y_{o}\right)^{t}\right)} \times \frac{\delta^{t+1}\left(\left(x_{o}, y_{o}\right)^{t+1}\right)}{\delta^{t+1}\left(\left(x_{o}, y_{o}\right)^{t}\right)}\right]^{1 / 2}$

De acordo com a equação (5), o índice é composto por quatro termos: dois correspondem aos períodos de tempo $t$ e $t+1, \delta^{t}\left(\left(x_{o}, y_{o}\right)^{t}\right)$ e $\delta^{t+1}\left(\left(x_{o}, y_{o}\right)^{t+1}\right)$, respectivamente; enquanto os outros dois $\delta^{t}\left(\left(x_{o}, y_{o}\right)^{t+1}\right)$ e $\delta^{t+1}\left(\left(x_{o}, y_{o}\right)^{t}\right)$ referem-se à comparação intertemporal.

O MI é interpretado da seguinte forma: (a) se MI > 1, indica aumento na produtividade; (b) se $\mathrm{MI}=1$, tem-se que a produtividade permaneceu constante; e (c) se MI < 1, houve uma redução de produtividade da DMU 37,38. Os índices de Malmquist foram calculados com base nas eficiências dos períodos $t$ e $t+1$, essas, por sua vez, obtidas pela equação (2).

\section{Fonte de dados e escolha do modelo}

A amostra da pesquisa foi constituída por 21 estados brasileiros e o Distrito Federal que atuaram 
no processo de transplante renal no Brasil. Nos modelos DEA, esses estados e o Distrito Federal são denominados DMU, os quais são avaliados como eficientes ou ineficientes.

Os anos de referência usados na pesquisa foram 2006 e 2011, por se tratarem de períodos antes e depois da instituição da Portaria no 2.600 29, de 21 de outubro de 2009, pelo Ministério da Saúde. Essa ação é denominada, aqui, de mudança institucional.

O critério de seleção da amostra foi a participação (via SUS) de cada estado e do Distrito Federal no sistema de transplante renal e a disponibilidade completa de informações. Os estados do Amapá, Mato Grosso, Rondônia, Roraima e Tocantins foram excluídos por conta da indisponibilidade de dados de input e output no período analisado.

Tendo em conta o modelo proposto por Marinho \& Cardoso 19 e Marinho 33 sobre eficiência no setor de transplantes, os inputs e outputs usados nessa pesquisa foram os seguintes:

- Inputs: os inputs são definidos como os gastos dos serviços hospitalares e dos serviços dos profissionais no sistema de transplante renal (por estado e pelo Distrito Federal). O somatório desses dois itens é correspondente ao gasto total no setor analisado;

- Output: o output é definido como o número de transplantes renais realizados por cada estado brasileiro e pelo Distrito Federal.

A coleta de dados foi realizada no site do Sistema de Informações Hospitalares (SIH) do SUS, usando-se os códigos dos procedimentos hospitalares (por local de internação) referente ao transplante renal. Para os anos de 2006 e 2011, os dados estão disponíveis (por estado) na homepage do Departamento de Informática do SUS (DATASUS; http://tabnet.datasus.gov.br/cgi/ deftohtm.exe?sih/cnv/qiuf.def, acessado em 11/Jun/2011). As informações utilizadas nesta pesquisa foram coletadas entre os meses de março e abril de 2012 e estão apresentadas na Tabela 1. Cabe ressaltar que o SUS não fornece dados relacionados com as quantidades de inputs, mas apenas o valor do gasto total referente a eles, que corresponde à soma dos gastos dos serviços hospitalares e dos profissionais.

Para que a metodologia DEA seja empregada, um modelo empírico foi desenvolvido, baseando-se em parâmetros de entrada (input) e de saída (output), conforme Figura 1. Nesse modelo, as DMU representam cada estado brasileiro e o Distrito Federal. As estimativas do modelo DEA-SBM e do MI foram realizadas por meio do software DEA Solver Professional versão 7.0 (SAITECH Inc., Holmdel, Estados Unidos).

\section{Resultados}

Na Tabela 2, verifica-se que, em média, no ano de 2006, os 21 estados brasileiros e o Distrito Federal (via SUS) gastaram com os serviços hospitalares e serviços de profissionais no setor de transplantes renais o equivalente a $\mathrm{R} \$ 1.891,52$ mil e $\mathrm{R} \$$ 377,87 mil, respectivamente. O gasto total médio desses estados e do Distrito Federal foi de R\$ 2.269,38 mil.

Ao comparar a média de custos de 2011 (Tabela 3) com 2006 (Tabela 2), tem-se um crescimento do valor médio de 91,2\% para gastos dos serviços hospitalares; $167,02 \%$ para gastos dos profissionais e $103,83 \%$ para os gastos totais com transplantes renais. Constata-se também que o número de transplantes renais realizados nos estados e no Distrito Federal, em média, apresentou uma tendência crescente, passando de aproximadamente 127, em 2006, para 201 transplantes em 2011 - uma expansão de 58,27\%.

Na Tabela 4, estão apresentados os escores de eficiência dos 21 estados brasileiros do Distrito Federal no sistema público de transplante renal, para os anos de 2006 e 2011. Em 2006, observase que cinco estados estavam localizados na fronteira de eficiência (ou seja, foram apontados como eficientes na maximização do número de transplantes) sendo eles: São Paulo, Acre, Paraná, Amazonas e Maranhão. Por sua vez, 16 estados e Distrito Federal ficaram abaixo dessa fronteira. Ademais, verifica-se que, desses 16 estados e Distrito Federal, o Pará, com um escore de eficiência de 0,85 , apresentou o pior resultado entre aqueles ineficientes.

No ano de 2011, ocorreu mudança na fronteira de eficiência desse sistema, e sete estados se tornam eficientes, com destaque para Sergipe, São Paulo, Alagoas, Paraná, Mato Grosso do Sul, Ceará e Maranhão. A quantidade de estados ineficientes tornou-se menor, correspondente a 14, somado o Distrito Federal. Além disso, para alguns deles houve melhora no grau de ineficiência; já para outros, a ineficiência sofreu uma expansão. Acre e Amazonas deixaram a fronteira de eficiência, atingido os patamares de eficiência de 0,94 e 0,98, respectivamente. O Pará foi o estado que se situou abaixo da fronteira (novamente) com o pior resultado em termos de eficiência, sendo seu score de 0,88. Ressalta-se que em ambos os períodos, São Paulo, Paraná e Maranhão sempre permaneceram na fronteira de eficiência (escore igual a 1).

A etapa seguinte consistiu em examinar, pelas folgas do modelo DEA-SBM (excessos de input ou escassez de output), as possíveis causas de ineficiência dos estados e do Distrito Federal que ficaram abaixo da fronteira de eficiência. 
Tabela 1

Relação dos 21 estados brasileiros e do Distrito Federal e variáveis insumos e produto para a metodologia Análise Envoltória de Dados (DEA), 2006 e 2011.

\begin{tabular}{|c|c|c|c|c|c|c|c|c|}
\hline \multirow[t]{3}{*}{ Estados } & \multicolumn{4}{|c|}{2006} & \multicolumn{4}{|c|}{2011} \\
\hline & \multicolumn{2}{|c|}{ Insumos } & \multirow[t]{2}{*}{ GT } & \multirow{2}{*}{$\begin{array}{l}\text { Produto } \\
\text { QTRenal }\end{array}$} & \multicolumn{2}{|c|}{ Insumos } & \multirow[t]{2}{*}{ GT } & \multirow{2}{*}{$\begin{array}{l}\text { Produto } \\
\text { QTRenal }\end{array}$} \\
\hline & GSH & GSP & & & GSH & GSP & & \\
\hline$A C$ & $27.660,46$ & $5.189,99$ & $32.850,45$ & 2 & $235.268,04$ & $75.882,40$ & $311.150,44$ & 16 \\
\hline$A L$ & $139.769,10$ & $29.064,30$ & $168.833,40$ & 10 & $190.678,14$ & $59.671,60$ & $250.349,74$ & 14 \\
\hline AM & $232.439,31$ & $49.306,29$ & $281.745,60$ & 19 & $253.666,98$ & $84.604,95$ & $338.271,93$ & 18 \\
\hline $\mathrm{BA}$ & $694.381,18$ & $135.979,07$ & $830.360,25$ & 47 & $1.332 .580,51$ & $370.451,86$ & $1.703 .032,37$ & 74 \\
\hline CE & $1.690 .888,93$ & $357.481,93$ & $2.048 .370,86$ & 115 & $3.339 .446,56$ & $1.065 .407,10$ & $4.404 .853,66$ & 208 \\
\hline DF & $489.220,48$ & $101.464,98$ & $590.685,46$ & 34 & $480.630,36$ & $147.615,09$ & $628.245,45$ & 30 \\
\hline ES & $816.928,15$ & $150.770,11$ & $967.698,26$ & 50 & $1.494 .434,84$ & $413.490,49$ & $1.907 .925,33$ & 82 \\
\hline GO & $1.065 .039,89$ & $216.165,82$ & $1.281 .205,71$ & 74 & $1.681 .686,11$ & $495.588,10$ & $2.177 .274,21$ & 100 \\
\hline MA & $366.055,47$ & $76.034,57$ & $442.090,04$ & 29 & $919.889,52$ & $299.183,28$ & $1.219 .072,80$ & 63 \\
\hline MS & $545.771,46$ & $106.915,21$ & $652.686,67$ & 37 & $337.638,09$ & $103.642,66$ & $441.280,75$ & 24 \\
\hline MG & $4.343 .492,08$ & $862.181,83$ & $5.205 .673,91$ & 284 & $8.499 .358,34$ & $2.343 .347,82$ & $10.842 .706,16$ & 459 \\
\hline PA & $650.164,34$ & $133.123,50$ & $783.287,84$ & 42 & $839.107,07$ & $252.028,50$ & $1.091 .135,57$ & 48 \\
\hline PB & $242.802,39$ & $48.786,48$ & $291.588,87$ & 17 & $471.449,10$ & $136.921,20$ & $608.370,30$ & 29 \\
\hline PR & $2.940 .763,83$ & $588.887,63$ & $3.529 .651,46$ & 213 & $5.900 .350,12$ & $1.696 .705,90$ & $7.597 .056,02$ & 358 \\
\hline PE & $1.745 .415,28$ & $322.559,79$ & $2.067 .975,07$ & 106 & $3.922 .452,87$ & $1.002 .015,50$ & $4.924 .468,37$ & 188 \\
\hline $\mathrm{PI}$ & $366.988,77$ & $65.913,59$ & $432.902,36$ & 23 & $541.467,59$ & $150.112,48$ & $691.580,07$ & 31 \\
\hline RJ & $1.013 .340,78$ & $226.547,57$ & $1.239 .888,35$ & 73 & $3.058 .770,03$ & $918.699,78$ & $3.977 .469,81$ & 183 \\
\hline RN & $228.379,45$ & $47.748,57$ & $276.128,02$ & 16 & $1.040 .866,11$ & $259.288,14$ & $1.300 .154,25$ & 51 \\
\hline RS & $4.013 .012,06$ & $759.502,83$ & $4.772 .514,89$ & 242 & $9.007 .545,86$ & $2.242 .791,01$ & $11.250 .336,87$ & 427 \\
\hline SC & $2.515 .850,44$ & $490.158,79$ & $3.006 .009,23$ & 154 & $4.901 .918,12$ & $1.392 .255,60$ & $6.294 .173,72$ & 273 \\
\hline SE & $144.455,01$ & $30.621,13$ & $175.076,14$ & 10 & $110.619,00$ & $35.749,88$ & $146.368,88$ & 7 \\
\hline $\mathrm{SP}$ & $17.340 .550,39$ & $3.508 .666,00$ & $20.849 .216,39$ & 1.197 & $31.005 .889,09$ & $8.651 .898,02$ & $39.657 .787,11$ & 1.736 \\
\hline
\end{tabular}

Fonte: Sistema Nacional de Transplantes, Departamento de Informática do SUS (http://snt.datasus.gov.br/SNT/index.jsf, acessado em 10/Jun/2011). GSH: gastos com serviços hospitalares; GSP: gastos com serviços profissionais; GT: gasto total com transplantes renais; QTRenal: quantidade de transplantes renais realizados.

Unidades Federativas: AC: Acre; AL: Alagoas; AM: Amazonas; BA: Bahia; CE: Ceará; DF: Distrito Federal; ES: Espírito Santo; GO: Goiás; MA: Maranhão; MG: Minas Gerais; MS: Mato Grosso do Sul; PA: Pará; PB: Paraíba; PE: Pernambuco; PI: Piauí; PR: Paraná; RJ: Rio de Janeiro; RN: Rio Grande do Norte; RS: Rio Grande do Sul; SC: Santa Catarina; SE: Sergipe; SP: São Paulo.

Na Tabela 5, observa-se que 12 estados (Bahia, Espírito Santo, Goiás, Minas Gerais, Pará, Paraíba, Pernambuco, Piauí, Rio de Janeiro, Rio Grande do Norte, Rio Grande do Sul e Santa Catarina) e o Distrito Federal estiveram abaixo da fronteira de eficiência nos dois períodos. Os estados Acre, Alagoas, Amazonas, Ceará, Mato Grosso do Sul e Sergipe variaram a posição entre a fronteira e abaixo dela.

A ineficiência dos estados Bahia, Espírito Santo, Minas Gerais, Paraíba, Pernambuco, Piauí, Rio Grande do Norte, Rio Grande do Sul e Santa Catarina, pelo lado do insumo em 2006 e 2011, deveu-se ao excesso dos gastos serviços hospitalares. Pelo lado do produto, a escassez de transplantes renais realizados foi o fator determinante para ineficiência nos dois períodos.
Em 2006, a ineficiência do Distrito Federal e dos estados Ceará, Goiás, Pará, Rio de Janeiro e Sergipe, pelo lado do input, ocorreu pelo excesso dos gastos serviços profissionais. Pelo lado do output, a escassez do número de transplantes renais realizados foi o motivo de não atingirem a fronteira. Em 2011, esses estados não apresentaram excesso de input, somente a escassez de output (Tabela 5).

Os estados do Acre e Amazonas foram ineficientes, em 2011, pelo lado do input, devido ao excesso dos gastos serviços profissionais e, pelo lado do output, a escassez de transplantes renais (Tabela 5). Por fim, Alagoas e Mato Grosso do Sul, em 2006, foram ineficientes pelo excesso dos gastos serviços hospitalares (lado do input) e escassez dos transplantes (lado do output). 
Figura 1

Modelo empírico do sistema.

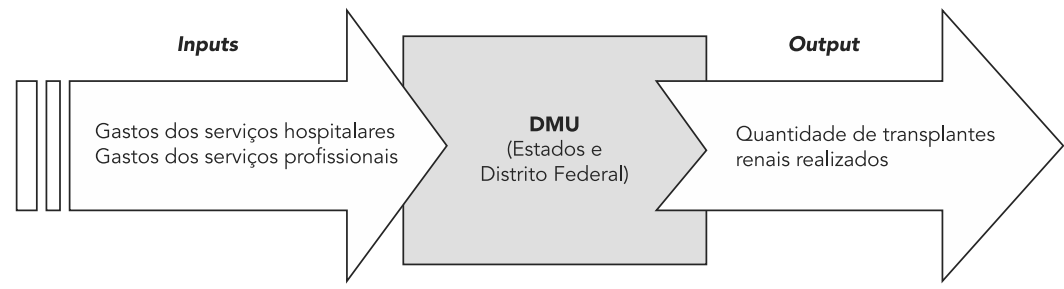

DMU: decision making units (unidades tomadoras de decisão).

\section{Tabela 2}

Estatística descritiva das variáveis consideradas na mensuração da eficiência dos 21 estados brasileiros e do Distrito Federa no sistema de transplante renal, 2006.

\begin{tabular}{|c|c|c|c|c|}
\hline \multirow[t]{2}{*}{2006} & \multicolumn{3}{|c|}{ Insumos } & \multirow{2}{*}{$\begin{array}{l}\text { Produto } \\
\text { QTRenal }\end{array}$} \\
\hline & GSH (R\$ mil) & GSP (R\$ mil) & GT (R\$ mil) & \\
\hline Média & $1.891,52$ & 377,87 & $2.269,38$ & 127 \\
\hline Desvio-padrão & $3.668,28$ & 740,22 & $4.408,37$ & 252 \\
\hline Coeficiente de variação (\%) & 193,93 & 195,89 & 194,25 & 198,43 \\
\hline Mínimo & 27,66 & 5,19 & 32,85 & 2 \\
\hline Máximo & $17.340,55$ & $3.508,67$ & $20.849,22$ & 1.197 \\
\hline Intervalo & $17.312,89$ & $3.503,48$ & $20.816,37$ & 1.195 \\
\hline
\end{tabular}

GSH: gastos com serviços hospitalares; GSP: gastos com serviços profissionais; GT: gasto total com transplantes renais;

QTRenal: quantidade de transplantes renais realizados.

Tabela 3

Estatística descritiva das variáveis consideradas na mensuração da eficiência dos 21 estados brasileiros e do Distrito Federal no sistema de transplante renal, 2011.

\begin{tabular}{|c|c|c|c|c|}
\hline \multirow[t]{2}{*}{2011} & \multicolumn{3}{|c|}{ Insumos } & \multirow{2}{*}{$\begin{array}{l}\text { Produto } \\
\text { QTRenal }\end{array}$} \\
\hline & GSH (R\$ mil) & GSP (R\$ mil) & GT (R\$ mil) & \\
\hline Média & $3.616,62$ & $1.008,97$ & $4.625,59$ & 201 \\
\hline Desvio-padrão & $6.662,68$ & $1.847,09$ & $8.508,67$ & 370 \\
\hline Coeficiente de variação (\%) & 184,22 & 183,07 & 183,95 & 184,08 \\
\hline Mínimo & 110,62 & 357,50 & 146,37 & 7 \\
\hline Máximo & $31.005,89$ & $8.651,90$ & $39.657,79$ & 1.736 \\
\hline Intervalo & $30.895,27$ & $8.616,1$ & $39.511,42$ & 1.729 \\
\hline
\end{tabular}

GSH: gastos com serviços hospitalares; GSP: gastos com serviços profissionais; GT: gasto total com transplantes renais; QTRenal: quantidade de transplantes renais realizados. 
Tabela 4

Escores de eficiência dos 21 estados brasileiros e do Distrito Federal no sistema público de transplante renal, 2006 e 2011.

\begin{tabular}{|c|c|c|c|c|c|}
\hline \multicolumn{3}{|c|}{2006} & \multicolumn{3}{|c|}{2011} \\
\hline Rank & Estados/Distrito Federal & Escore & Rank & Estados/Distrito Federal & Escore \\
\hline 1 & São Paulo & 1,00 & 1 & Sergipe & 1,00 \\
\hline 1 & Acre & 1,00 & 1 & São Paulo & 1,00 \\
\hline 1 & Paraná & 1,00 & 1 & Alagoas & 1,00 \\
\hline 1 & Amazonas & 1,00 & 1 & Paraná & 1,00 \\
\hline 1 & Maranhão & 1,00 & 1 & Mato Grosso do Sul & 1,00 \\
\hline 6 & Rio de Janeiro & 0,97 & 1 & Ceará & 1,00 \\
\hline 7 & Goiás & 0,94 & 1 & Maranhão & 1,00 \\
\hline 8 & Minas Gerais & 0,93 & 8 & Amazonas & 0,98 \\
\hline 9 & Bahia & 0,93 & 9 & Rio de Janeiro & 0,96 \\
\hline 10 & Ceará & 0,93 & 10 & Goiás & 0,95 \\
\hline 11 & Mato Grosso do Sul & 0,92 & 11 & Minas Gerais & 0,94 \\
\hline 12 & Piauí & 0,91 & 12 & Acre & 0,94 \\
\hline 13 & Paraíba & 0,90 & 13 & Paraíba & 0,94 \\
\hline 14 & Pernambuco & 0,90 & 14 & Santa Catarina & 0,93 \\
\hline 15 & Distrito Federal & 0,90 & 15 & Bahia & 0,93 \\
\hline 16 & Espírito Santo & 0,90 & 16 & Espírito Santo & 0,92 \\
\hline 17 & Rio Grande do Sul & 0,89 & 17 & Piauí & 0,92 \\
\hline 18 & Alagoas & 0,89 & 18 & Rio Grande do Sul & 0,92 \\
\hline 19 & Rio Grande do Norte & 0,87 & 19 & Distrito Federal & 0,91 \\
\hline 20 & Santa Catarina & 0,87 & 20 & Rio Grande do Norte & 0,90 \\
\hline 21 & Sergipe & 0,86 & 21 & Pernambuco & 0,89 \\
\hline 22 & Pará & 0,85 & 22 & Pará & 0,88 \\
\hline
\end{tabular}

Na Tabela 6, estão apresentados os resultados do MI e de suas decomposições (efeito emparelhamento e deslocamento da fronteira). Para o período 2006-2011, o Estado de Alagoas foi o único que conseguiu aumentar sua produtividade, com índice maior que a unidade $(1,05)$. Vinte (20) estados e o Distrito Federal apresentaram redução de produtividade, com o valor do índice menor do que 1 .

Examinando a decomposição do MI, tem-se que 17 estados e o Distrito Federal aumentaram o índice de mudança pura de eficiência (efeito emparelhamento) com valores maiores que 1 . A redução de eficiência (índice menor que 1) foi constatada em quatro estados, sendo eles: Acre, Amazonas, Rio de Janeiro e São Paulo.

Observa-se que a perda de produtividade de grande parte dos estados e do Distrito Federal deveu-se ao índice de mudança da escala de eficiência (efeito deslocamento de fronteira). De acordo com os resultados, 20 estados e o Distrito Federal apresentaram um índice menor que 1, indicando um deslocamento da fronteira de produção para um nível inferior. O Estado de São
Paulo foi o único, nesse período, que apresentou aumento na produvidade, em razão das mudanças institucionais.

\section{Discussão}

Os resultados desta pesquisa sugerem que o processo de transplantes renais tem-se apresentado uma atividade com grande variabilidade entre os estados e o Distrito Federal, uma vez que os altos desvios-padrão apontam para disparidades na gestão dos recursos aplicados nesse setor. No contexto atual, os estados das regiões Sul e Sudeste realizam as atividades de captação e transplantes de órgãos com maior eficiência do que os estados das regiões Nordeste, Centro-oeste e Norte. A oferta do órgão rim torna-se insuficiente nesses locais para suprir a demanda, gerando as filas de espera por um transplante renal 1,11,42.

De acordo com os resultados dos escores de eficiência de cada estado e do Distrito Federal, pode-se afirmar que houve mudança na fronteira de eficiência de 2006 para 2011. Além disso, 
Folgas dos 21 estados brasileiros e do Distrito Federal no sistema público de transplante renal, 2006 e 2011.

\begin{tabular}{|c|c|c|c|c|c|c|c|c|}
\hline \multirow{3}{*}{$\begin{array}{l}\text { Estados/ } \\
\text { Distrito } \\
\text { Federal }\end{array}$} & \multicolumn{4}{|c|}{2006} & \multicolumn{4}{|c|}{2011} \\
\hline & \multirow[t]{2}{*}{ Escore } & \multicolumn{2}{|c|}{ Excesso } & \multirow{2}{*}{$\begin{array}{l}\text { Escassez } \\
\text { OTRenal }\end{array}$} & \multirow[t]{2}{*}{ Escore } & \multicolumn{2}{|c|}{ Excesso } & \multirow{2}{*}{$\begin{array}{l}\text { Escassez } \\
\text { QTRenal }\end{array}$} \\
\hline & & GSH & GSP & & & GSH & GSP & \\
\hline$A C$ & 1,00 & 0,00 & 0,00 & 0,00 & 0,94 & 0,00 & $2.869,31$ & 1,03 \\
\hline$A L$ & 0,89 & $1.289,01$ & 0,00 & 1,20 & 1,00 & 0,00 & 0,00 & 0,00 \\
\hline AM & 1,00 & 0,00 & 0,00 & 0,00 & 0,98 & 0,00 & $6.086,81$ & 0,29 \\
\hline BA & 0,93 & $27.382,58$ & 0,00 & 3,51 & 0,93 & $63.289,04$ & 0,00 & 5,94 \\
\hline CE & 0,93 & 0,00 & $17.555,38$ & 8,68 & 1,00 & 0,00 & 0,00 & 0,00 \\
\hline DF & 0,90 & 0,00 & 897,32 & 3,80 & 0,91 & 0,00 & 0,00 & 3,01 \\
\hline ES & 0,90 & $75.673,16$ & 0,00 & 5,81 & 0,92 & $74.859,63$ & 0,00 & 6,96 \\
\hline GO & 0,94 & 0,00 & 901,38 & 4,95 & 0,95 & 0,00 & 0,00 & 5,69 \\
\hline MA & 1,00 & 0,00 & 0,00 & 0,00 & 1,00 & 0,00 & 0,00 & 0,00 \\
\hline MS & 0,92 & $24.683,98$ & 0,00 & 3,08 & 1,00 & 0,00 & 0,00 & 0,00 \\
\hline MG & 0,93 & $54.893,71$ & 0,00 & 21,10 & 0,94 & $264.882,34$ & 0,00 & 27,12 \\
\hline PA & 0,85 & 0,00 & 497,63 & 7,30 & 0,88 & 0,00 & 0,00 & 6,78 \\
\hline PB & 0,90 & $12.775,97$ & 0,00 & 1,80 & 0,94 & $17.607,88$ & 0,00 & 1,98 \\
\hline PR & 1,00 & 0,00 & 0,00 & 0,00 & 1,00 & 0,00 & 0,00 & 0,00 \\
\hline $\mathrm{PE}$ & 0,90 & $141.713,80$ & 0,00 & 11,45 & 0,89 & $447.846,15$ & 0,00 & 24,35 \\
\hline $\mathrm{PI}$ & 0,91 & $51.528,64$ & 0,00 & 2,21 & 0,92 & $41.564,61$ & 0,00 & 2,74 \\
\hline RJ & 0,97 & 0,00 & $21.581,02$ & 2,26 & 0,96 & 0,00 & 0,00 & 7,06 \\
\hline $\mathrm{RN}$ & 0,87 & $3.170,79$ & 0,00 & 2,40 & 0,90 & $159.739,88$ & 0,00 & 5,63 \\
\hline RS & 0,89 & $230.806,81$ & 0,00 & 28,50 & 0,92 & $1.136 .040,84$ & 0,00 & 39,19 \\
\hline SC & 0,87 & $70.741,19$ & 0,00 & 23,58 & 0,93 & $64.657,84$ & 0,00 & 21,17 \\
\hline SE & 0,86 & 0,00 & 269,63 & 1,70 & 1,00 & 0,00 & 0,00 & 0,00 \\
\hline SP & 1,00 & 0,00 & 0,00 & 0,00 & 1,00 & 0,00 & 0,00 & 0,00 \\
\hline
\end{tabular}

GSH: gastos com serviços hospitalares; GSP: gastos com serviços profissionais; GT: gasto total com transplantes renais; QTRenal: quantidade de transplantes renais realizados.

Unidades Federativas: AC: Acre; AL: Alagoas; AM: Amazonas; BA: Bahia; CE: Ceará; DF: Distrito Federal; ES: Espírito Santo; GO: Goiás; MA: Maranhão; MG: Minas Gerais; MS: Mato Grosso do Sul; PA: Pará; PB: Paraíba; PE: Pernambuco; PI: Piauí; PR: Paraná; RJ: Rio de Janeiro; RN: Rio Grande do Norte; RS: Rio Grande do Sul; SC: Santa Catarina; SE: Sergipe; SP: São Paulo.

os estados e o Distrito Federal classificados como ineficientes não apresentaram elevado grau de ineficiência, sendo o menor valor de 0,85 em 2006 e 0,88 em 2011. Examinando as causas de ineficiência produtiva, foi possível constatar que, pelo lado dos inputs, a maior parte dos estados não atingiu a fronteira pelo excesso dos gastos com serviços hospitalares. Já outros estados e o Distrito Federal, pelo excesso dos gastos com profissionais. Isso mostra que a utilização desses recursos financeiros não implicou necessariamente melhoria no sistema. Pelo lado do output, a escassez do número de transplantes renais impactou de forma negativa o desempenho dos estados e do Distrito Federal.

Os índices de produtividade corroboraram os escores de eficiência obtidos pelos estados e pelo Distrito Federal, ao mostrar que após as mudanças institucionais implementadas pelo Ministé- rio da Saúde (Portaria no 2.600 29), a maioria dos estados e o Distrito Federal (com exceção de São Paulo) não apresentaram avanços de produtividade ao longo do tempo, apenas mudança pura de eficiência. Assim, mesmo o SNT atuando na busca da melhoria contínua nesse sistema, ainda se tornam persistentes os problemas de gestão e gerenciamento do setor.

O sistema de transplantes renais no Brasil, no período atual, apresenta um número de cirurgias que está abaixo das necessidades da população, em virtude, sobretudo, da falta de doações efetivas desse órgão 2,3,8,21. O funcionamento adequado do processo captação de órgãos, o empenho e o esforço das equipes de transplantes atuando na questão doação-transplante é fundamental para que todo o processo ocorra de maneira eficiente em todos os estados brasileiros. Logo, faz-se necessário oferecer mecanismos de incentivos às 
Decomposição do índice de Malmquist para os 21 estados brasileiros e o Distrito Federal, 2006 e 2011.

\begin{tabular}{|c|c|c|c|}
\hline $\begin{array}{l}\text { Estados Estados/ } \\
\text { Distrito Federal }\end{array}$ & $\begin{array}{l}\text { Emparelhamento } \\
\text { (catch-up) }\end{array}$ & $\begin{array}{c}\text { Deslocamento da fronteira } \\
\text { (frontier-shift) }\end{array}$ & $\begin{array}{l}\text { Índice de } \\
\text { Malmquist }\end{array}$ \\
\hline$A C$ & 0,94 & 0,94 & 0,88 \\
\hline$A L$ & 1,22 & 0,86 & 1,05 \\
\hline AM & 0,95 & 0,73 & 0,69 \\
\hline BA & 1,03 & 0,69 & 0,71 \\
\hline CE & 1,08 & 0,73 & 0,79 \\
\hline DF & 1,01 & 0,74 & 0,75 \\
\hline ES & 1,12 & 0,67 & 0,76 \\
\hline GO & 1,01 & 0,72 & 0,73 \\
\hline MA & 1,04 & 0,73 & 0,76 \\
\hline MS & 1,17 & 0,71 & 0,83 \\
\hline MG & 1,03 & 0,70 & 0,72 \\
\hline PA & 1,03 & 0,73 & 0,75 \\
\hline PB & 1,08 & 0,68 & 0,74 \\
\hline PR & 1,01 & 0,71 & 0,72 \\
\hline$P E$ & 1,06 & 0,65 & 0,69 \\
\hline PI & 1,15 & 0,66 & 0,76 \\
\hline RJ & 0,99 & 0,75 & 0,75 \\
\hline RN & 1,05 & 0,64 & 0,67 \\
\hline $\mathrm{RS}$ & 1,08 & 0,65 & 0,70 \\
\hline SC & 1,10 & 0,70 & 0,77 \\
\hline SE & 1,17 & 0,82 & 0,96 \\
\hline SP & 0,04 & 5,83 & 0,25 \\
\hline
\end{tabular}

equipes de busca ativa de órgãos para garantir melhorias significativas no desempenho do sistema 42,43,44,45.

O Estado de São Paulo foi destaque nos resultados desta pesquisa, exibindo grande número de transplantes renais, associada a um bom desempenho de eficiência. Tal fato pode estar relacionado às questões econômicas e sociais de que o estado dispõe; pela gestão do setor e pela existência das OPO, que contribuem para o ótimo funcionamento do processo de captação de órgãos e tecidos. Além disso, esse estado se destaca pela atuação positiva em relação aos reduzidos tempos de espera para transplantes 2 .

A questão da ineficiência do Estado de Santa Catarina merece ser analisado aqui com maior ênfase. Santa Catarina foi classificada como ineficiente na análise da fronteira de eficiência e de produtividade ao longo do tempo, considerando os insumos e produtos dessa pesquisa. Contudo, é importante ressaltar que, em 2011, a taxa de transplantes desse estado foi de 25 doadores efetivos por milhão de habitantes - o melhor resultado já obtido por um estado brasileiro. Esse fato está relacionado aos incentivos dos setores públicos locais (governo estadual), da solução de pequenas barreiras na logística do processo de identificação do doador até a realização do transplante e da relação harmônica que existe entre as equipes e a coordenação de transplante local 46 . Por conseguinte, devido à indisponibilidade de informações sobre outros insumos usados pelo SUS nesse sistema, não se pode afirmar se realmente esse estado é ineficiente.

Por outro lado, Rio de Janeiro e Bahia podem ser classificados como estados ineficientes, pois apresentam baixa taxa de doação efetiva de órgãos por causa dos seguintes problemas: dificuldades para a efetivação de transplantes mesmo para os inscritos em filas de espera de transplante de rim e com compatibilidade genética; falta de exame de sangue atualizado; inexistência ou não de revalidação de exames pré-transplante e inconsistência de números telefônicos para rápida localização dos selecionados 8 .

De maneira geral, esse quadro desigual de acesso aos transplantes no país ocorre em virtude de vários fatores, tais como: (a) falta de iden- 
tificação e exames para confirmação de morte encefálica; (b) cuidados intensivos com os potenciais doadores; (c) capacitação inadequada dos profissionais no processo de abordagem das famílias para doação; (d) funcionamento não adequado das centrais estaduais de transplantes (isto é, desigualdades na capacidade operacional dessas centrais); (e) regras nacionais não observadas (como, por exemplo, vinculação das equipes a centros transplantadores; distribuição adequada de imunossupressores; encaminhamento de órgãos não aproveitados para estados próximos; execução da tipagem HLA de toda a lista de espera de rim), que geram prejuízos aos pacientes; (f) comissões intra-hospitalares não ativas; (g) dificuldades em se realizar os exames pré-transplante pelo SUS, gerando problemas de acesso a esses tratamentos para a população de baixa renda e residentes que estão distantes dos centros transplantadores; e (h) médicos intensivistas sobrecarregados, entre outros 47,48.

No Brasil, Marinho \& Cardoso 19 e Marinho 33 desenvolveram, por meio da metodologia DEA, uma análise da eficiência técnica e de escala do SNT, no período de 1995 a 2003. De acordo com seus resultados, verificou-se uma tendência à redução da eficiência do SNT, com uma rápida recuperação entre 2001 e 2003. Além disso, constataram uma expansão da eficiência nos transplantes de fígado; todavia, para os rins, a eficiência não ficou bem definida. Os autores concluíram que o SNT programou medidas eficazes de ajus- tes para o problema das filas de transplantes de órgãos no Brasil apenas no curto prazo.

Ozcan ey al. 4 utilizaram a DEA para mensurar a eficiência técnica das OPO nos Estados Unidos. A unidade de análise considerada foi cada OPO, formando uma amostra (em 1995) correspondente a 64 unidades. Conforme os resultados, seis das 22 maiores OPO foram classificadas como ineficientes, e 23 das 42 menores foram vistas como ineficientes. Os autores destacaram a necessidade de investigar a questão da eficiência no setor, objetivando melhorar os resultados da análise e, assim, contribuir com um elevado número de indivíduos que esperam por um transplante.

A análise de eficiência dos estados brasileiros e do Distrito Federal proposta neste estudo indica a necessidade de uma melhor alocação e/ou aplicação dos recursos gastos pelo SUS na área de transplantes renais. Com base nisso, o Ministério da Saúde pode tentar coordenar e planejar ainda mais suas decisões operacionais e administrativas no processo de doação de órgãos. Porém, vale ressaltar as restrições que existem no país referentemente às informações sobre os inputs e outputs desse setor, bem como sobre indicadores de qualidade para os transplantes e de sobrevida esperada dos transplantados. Tais dados são escassos e/ou não divulgados pelo SNT, gerando dificuldades no desenvolvimento de análises e políticas públicas no setor.

\section{Resumen}

El objetivo fue evaluar la eficacia de los estados brasileños y en el Distrito Federal en el trasplante renal público y su productividad entre 2006 y 2011. Se utilizó el Análisis de Involucrados de Datos (DEA) y el índice de Malmquist. La muestra estuvo conformada por 22 estados. Las entradas corresponden a los gastos totales de trasplantes de riñón, referidos a servicios generales y hospitalarios de los profesionales de este sector. Se utilizó el número de riñones trasplantados como punto de salida. Entre los estados existe una discrepancia significativa, en relación con la captación y el número de trasplantes de riñón. Es evidente la ineficiencia del sistema, que puede ser causada por la gestión de operaciones inadecuadas, el incumplimiento de las normas nacionales, los comités no hospitalarios y personal hospitalario activo sobrecargado. Sin embargo, su productividad ha mejorado con los cambios institucionales. Por lo tanto, los estados que participaron en el proceso de recepción y donación de riñones para trasplantes presentan ineficiencia en términos de gastos administrativos yoperativos.

Trasplante de Riñón; Gestión en Salud; Sistema Único de Salud 


\section{Colaboradores}

C. K. F. Costa contribuiu com a concepção do projeto, estimação, análise e interpretação dos dados; redação do artigo e correções e aprovação final da versão a ser publicada. G. Balbinotto Neto colaborou na concepção, projeto, elaboração do artigo, análise, correções e aprovação final da versão a ser publicada. L. M. B. Sampaio participou da concepção do projeto, estimação, correções e aprovação final da versão a ser publicada.

\section{Referências}

1. Marinho A, Cardoso SS, Almeida VV. Os transplantes de órgãos nos estados brasileiros. Rio de Janeiro: Instituto de Pesquisa Econômica Aplicada; 2007. (Texto para Discussão, 1317).

2. Medina-Pestana JO, Galante NZ, Tedesco-Silva Jr. H, Garcia VD, Abbud-Filho M, Campos HH, et al. O contexto do transplante renal no Brasil e sua disparidade geográfica. J Bras Nefrol 2011; 33:472-84.

3. Garcia GG, Harden P, Chapman J. O papel global do transplante renal. J Bras Nefrol 2012; 34:1-7.

4. Ozcan YA, Begun JW, McKinney MM. Benchmarking organ procurement organizations: a national study. Health Serv Res 1999; 34:855-74.

5. The U.S. Organ Procurement and Transplantation Network. Scientific registry of transplant recipients: annual report 2009. http://optn.transplant. hrsa.gov/ar2009/ar_archives.htm (acessado em 12/Abr/2010).

6. The U.S. Organ Procurement and Transplantation Network. Scientific registry of transplant recipients: annual report 2010. http://optn.transplant. hrsa.gov/ar2009/ar_archives.htm (acessado em 12/Ago/2011).

7. Associação Brasileira de Transplantes de Órgãos. Inserção do Brasil no mundo. Registro Brasileiro de Transplantes 2012; XVIII:3.

8. Garcia VD. Vamos levando.... Registro Brasileiro de Transplantes 2011; XVII:3.

9. Diretoria e Conselho da Associação Brasileira de Transplantes de Órgãos. Transplantes 2011. Registro Brasileiro de Transplantes 2011; XVII: 3.

10. Marinho A. Um estudo sobre as filas para transplantes no Sistema Único de Saúde brasileiro. Cad Saúde Pública 2006; 22:2229-39.

11. Marinho A, Cardoso SS, Almeida VV. Desigualdade de transplantes de órgãos no Brasil: análise do perfil dos receptores por sexo e raça ou cor. Rio de Janeiro: Instituto de Pesquisa Econômica Aplicada; 2011. (Texto para Discussão, 1629).

12. Garcia VD, Abbud Filho M, Neumann J, Pestana JOM. Transplante de órgãos e tecidos. 2a Ed. São Paulo: Editora Segmento Farma; 2006.
13. Garcia VD, Abbud Filho M, Campos HH, Pestana JOM. Política de transplantes no Brasil. In: Garcia VD, Abbud Filho M, Neumann J, Pestana JOM, organizadores. Transplante de órgãos e tecidos. 2a Ed. São Paulo: Editora Segmento Farma; 2006. p. 43-9.

14. Garcia VD, Almeida TAM, Pinto JBT. Processo de doação-transplante. In: Garcia VD, Abbud Filho M, Neumann J, Pestana JOM, organizadores. Transplante de órgãos e tecidos. 2a Ed. São Paulo: Editora Segmento Farma; 2006. p. 115-27.

15. Marinho A. Um estudo sobre as filas para internações e para transplantes no sistema único de saúde brasileiro. Rio de Janeiro: Instituto de Pesquisa Econômica Aplicada; 2004. (Texto para Discussão, 1055).

16. Marinho A, Cardoso SS, Almeida VV. Disparidade nas filas para transplantes de órgãos nos estados brasileiros. Cad Saúde Pública 2010; 26:786-96.

17. Silva EN. Ensaios em economia da saúde: transplantes de rim [Tese de Doutorado]. Porto Alegre: Faculdade de Ciências Econômicas, Universidade Federal do Rio Grande do Sul; 2008.

18. Dor A, Pauly MV, Eichleay MA, Held PJ. End-stage renal disease and economic incentives:the international study of health care organization and financing. Cambridge: National Bureau of Economic Research; 2007. (Working Paper, 13125).

19. Marinho A, Cardoso SS. Avaliação da eficiência técnica e da eficiência de escala do sistema nacional de transplantes. Rio de Janeiro: Instituto de Pesquisa Econômica Aplicada; 2007. (Texto para Discussão, 1260).

20. Godoy MR, Balbinotto Neto G, Ribeiro EP. Earnings and chronic renal disease. Transplant Proc 2007; 39:378-80.

21. Costa CKF. Ensaios sobre a economia dos transplantes renais no Brasil: incentivos e eficiência [Tese de Doutorado]. Porto Alegre: Faculdade de Ciências Econômicas, Universidade Federal do Rio Grande do Sul; 2012. 
22. Mendes FDR. O sistema nacional de transplantes. In: Manfro RC, Noronha IL, Silva Filho AP, organizadores. Manual de transplante renal. Porto Alegre: Edições Manole; 2004. p. 372-85.

23. Brasil. Lei no 9.434, de 4 de fevereiro de 1997. Dispõe sobre a remoção de órgãos, tecidos e partes do corpo humano para fins de transplante e tratamento e dá outras providências. Diário Oficial da União 1997; 5 fev.

24. Brasil. Decreto no 2.268, de 30 de junho de 1997. Regulamenta a Lei no 9.434, de 4 de fevereiro de 1997, que dispõe sobre a remoção de órgãos, tecidos e partes do corpo humano para fim de transplante e tratamento, e dá outras providências. Diário Oficial da União 1997; 1 jul.

25. Gediel JAP. Os transplantes de órgãos e a invenção moderna do corpo. Curitiba: Editora Moinho do Verbo; 2000

26. Brasil. Lei no 10.211, de 23 de março de 2001. Altera dispositivos da Lei no 9.434, de 4 de fevereiro de 1997, que "dispõe sobre a remoção de órgãos, tecidos e partes do corpo humano para fins de transplante e tratamento". Diário Oficial da União 2001; 24 mar.

27. Brasil. Lei no 11.521 , de 18 de setembro de 2007. Altera a Lei no 9.434, de 4 de fevereiro de 1997, para permitir a retirada pelo Sistema Único de Saúde de órgãos e tecidos de doadores que se encontrem em instituições hospitalares não autorizadas a realizar transplantes. Diário Oficial da União 2007; 19 set.

28. Tribunal de Contas da União. Relatório de avaliação de programa: Programa Doação, Captação e Transplante de Órgãos e Tecidos; 2006. http:// portal2.tcu.gov.br/portal/pls/portal/docs/690420. PDF (acessado em 12/Mai/2010).

29. Brasil. Portaria oo 2.600 de 21 de outubro de 2009. Aprova o Regulamento Técnico do Sistema Nacional de Transplantes. Diário Oficial da União 2009; 21 out.

30. Brasil. Portaria no 1.752 de 23 de setembro de 2005. Determina a constituição de Comissão Intra-Hospitalar de Doação de Órgãos e Tecidos para Transplante em todos os hospitais públicos, privados e filantrópicos com mais de 80 leitos. Diário Oficial da União 2005; 23 set.

31. Ministério da Saúde. Número de transplantes no Brasil mais do que dobra em 10 anos. http://portalsaude.saude.gov.br/portalsaude/no ticia/4234/162/numero-de-transplantes-no-bra sil-mais-que-dobra-em-dez-anos.html (acessado em 28/Mai/2012)

32. Ministério da Saúde. Relação entre a lista de espera de 2010 e 2011. http://189.28.128.35/portal/ public/transplantes/areaEscpecializada/Relato rioEstatistico/lista_de_espera (acessado em 26/ Mai/2012).

33. Marinho A. Estado de uma nação: texto de apoio. A situação dos transplantes de órgãos no Brasil. Rio de Janeiro: Instituto de Pesquisa Econômica Aplicada; 2009. (Texto para Discussão, 1389).

34. Jacobs R, Smith PC, Street A. Measuring efficiency in health care analytic techniques and health policy. Cambridge: Cambridge University Press; 2006.
35. Charnes A, Cooper WW, Rhodes E. Measuring the efficiency of decision making units. Eur J Oper Res 1978; 2:429-44.

36. Banker RD, Charnes A, Cooper WW. Some models for estimating technical and scale inefficiencies in data envelopment analysis. Manage Sci 1984; 30:1078-93.

37. Cooper WW, Seiford LM, Tone K. Data envelopment analysis: a comprehensive text with models, applications, references and DEA-solver software. 2nd Ed. New York: Springer; 2007.

38. Ferreira CMC, Gomes AP. Introdução à análise envoltória de dados: teoria, modelos e aplicações. Viçosa: Editora UFV; 2009.

39. Tone K. DEA with controllable category levels. In: Proceedings of the Spring National Conference of the Operations Research Society of Japan. Tokyo: Tokyo Institute of Technology; 1997; p. 126-7.

40. Tone K. A slacks-based measure of efficiency in data envelopment analysis. Eur J Oper Res 2001; 130:498-509.

41. Färe R, Grosskoff S, Norris M, Zhang Z. Productivity growth, technical progress, and efficiency change in industrialized countries. Am Econ Rev 1994; 84:66-83.

42. Garcia VD, Garcia RL. Avaliação do sistema de transplantes renais no Estado do Rio Grande do Sul/Brasil: uma abordagem de agente-principal. Medware 2010; (8). http://www.mednet.cl/link. cgi/Medwave/EstudiosOriginales/4712 (acessado em 09/Ago/2010).

43. Costa CKF, Balbinotto Neto G, Sampaio LMB. Transplantes renais no Brasil: uma abordagem da Teoria da Agência. In: Anais do XVIII Fórum Banco do Nordeste de Desenvolvimento - XVII Encontro Regional de Economia. http://www.bnb.gov. $\mathrm{br} /$ content/aplicacao/eventos/forumbnb2012/ docs/sim_3_mesa5_transplantes_renais_brasil_ abordagem_teoria_agencia.pdf (acessado em 27/ Set/2013).

44. Balbinotto Neto G, Costa CKF. Kidney transplants: an approach from the agency theory applied to Brazil. Transplantation 2012; 94:807.

45. Smith PC. Measuring health system performance. Eur J Health Econ 2002; 3:145-8.

46. Associação Brasileira de Transplantes de Órgãos. Entrevista. Boletim Informativo da Associação Brasileira de Transplante de Órgãos 2011; 14:19-21.

47. Mattia ALD, Rocha ADM, Freitas Filho JPA, Barbosa MH, Rodrigues MB, Oliveira MG. Análise das dificuldades no processo de doação de órgãos: uma visão integrativa da literatura. Revista Bioethikos 2010; 4:66-74

48. Claussel NO, Gonçalves LFS, Veronese FJV. Manutenção de doadores de órgãos. In: Barreto SM, Vieira SRR, Pinheiro CTS, organizadores. Rotinas em terapia intensiva. 3a Ed. Porto Alegre: Editora Artmed; 2001. p. 543-9.

Recebido em 26/Jun/2013

Versão final reapresentada em 14/Jan/2014

Aprovado em 30/Jan/2014 